\title{
LA SEGURIDAD INTEGRAL EN ECUADOR: UNA VISIÓN CRÍTICA DEL CONCEPTO A UNA DÉCADA DE SU CONCEPCIÓN
}

\author{
Lester Cabrera ${ }^{1}$ \\ FLACSO
}

\section{Resumen:}

El presente artículo tiene como objetivo presentar un panorama crítico sobre la evolución del concepto de seguridad integral, a una década de su generación, tomando como aspecto clave el criterio políticoideológico de contar con un nuevo concepto de seguridad, y las implicancias de dicho cambio. En este sentido, se realiza un análisis sobre los diferentes documentos, tanto legales como orientadores, en los que se establecen parámetros para su institucionalización y aplicación, relacionando esto con el contexto que influyó en los eventuales cambios del mismo. Se concluye que el establecimiento del concepto no fue adecuado, considerando el contexto interno y externo de Ecuador. Lo anterior, sumado a una amplitud en sus alcances y límites, y un bajo nivel de comprensión y ejecución, generó una disminución de las capacidades institucionales del país en otorgar seguridad.

Palabras Clave: Seguridad integral, Ecuador, ideología, instituciones

Title in English: Integral Security in Ecuador: A critical vision of the concept after a decade of its conception

\section{Abstract:}

The objective of this article is to present a critical panorama on the evolution of the concept of integral security, a decade after its generation, taking as a key aspect the political-ideological criteria of having a new concept of security, and the implications of such change. In this sense, it is analyzed different documents, both legal and guidance, in which parameters are established for their institutionalization and application, relating this to the context that influenced the eventual changes. It is concluded that the establishment of the concept was not adequate, considering the internal and external context of Ecuador. Therefore, coupled with a breadth in its scope and limits, and a low level of understanding and execution, generated a decrease in the country's institutional capacities in granting security.

Key Words: Integral security, Ecuador, ideology, institutions

Copyright (C) UNISCI, 2019.

Las opiniones expresadas en estos artículos son propias de sus autores, y no reflejan necesariamente la opinión de UNISCI. The views expressed in these articles are those of the authors, and do not necessarily reflect the views of UNISCI.

\footnotetext{
${ }^{1}$ Lester Cabrera es Profesor Invitado, Departamento de Estudios Internacionales y Comunicación, FLACSO (Ecuador)

E-mail: <lecabrerafl@flacso.edu.ec>

DOI: http://dx.doi.org/10.31439/UNISCI-69
} 


\section{Introducción}

¿Cómo ha sido la evolución del concepto de seguridad integral, tomando en consideración lo señalado en los diferentes documentos legales como orientadores que se han elaborado? ¿Cuáles son los elementos críticos del concepto de seguridad integral, y sus consecuencias en el ámbito seguritario del Ecuador? El presente trabajo tiene como objetivo responder a las anteriores interrogantes. Pero sin perjuicio de un posterior desarrollo de las mismas, resulta metodológicamente adecuado dar respuestas simples, de carácter introductorio. El concepto de seguridad integral ha sido uno de los elementos más representativos de la administración de Rafael Correa en Ecuador, el cual fue establecido como una forma de incorporar "otros" elementos a la visión clásica de la seguridad del país, pero también como una manera de establecer un quiebre con un pasado, considerando que el propio proyecto político poseía aspectos refundacionales del Estado. Pero dentro de los 10 años de gestión, el concepto posee múltiples visiones y alcances, que no permiten una adecuada comprensión del mismo, lo que se visualiza no solamente en las diversas concepciones del mismo en los instrumentos y lineamientos como en los planes gubernamentales relacionados, sino que en la acción de los organismos encargados de ejecutar y plasmar el mencionado concepto a la realidad.

Uno de los aspectos a considerar, es que derivado de la amplitud, tanto en el concepto en sí como en los procesos que implicaban su aplicación dentro de la seguridad del país, generó una serie de consecuencias dentro del plano seguritario del Ecuador. En este sentido, la amplitud del concepto de seguridad integral, y su vinculo con otros aspectos y actores, que no guardan una relación directa con la seguridad, dio como resultado un proceso de desmilitarización y debilitamiento doctrinario, específicamente en aquellas instituciones que, tradicionalmente, han sido parte del resguardo del país frente a amenazas. Aquello generó a su vez, que el país descuidara una parte esencial de su seguridad tradicional, buscando adaptarse a la nueva noción doctrinaria de la seguridad, pero sin una guía adecuada que permitiese dicho cambio, dentro de tiempos razonables y tomando en cuenta a las nuevas instituciones que debían ser parte de la ejecución de este nuevo concepto de seguridad integral.

Este estudio se plantea analizar el concepto de seguridad integral, tomando como base las definiciones, elementos y alcances que posee dicho concepto, en cada uno de las leyes y documentos orientadores que se han elaborado, conocidos con Planes Nacionales de Seguridad Integral. Aquello se efectuará considerando un análisis tanto del texto como de la eventual influencia del contexto, con el objetivo de establecer un grado de evolución de la seguridad en el país, como también en las formas que el concepto se ha ido incorporando en la institucionalidad. En este sentido, se observa como una limitante el desconocimiento que tanto autoridades como académicos vinculados a la temática poseen sobre el mismo, generando como consecuencia una fuerte incertidumbre en las implicancias que la aplicación del mismo posea en instituciones que no necesariamente pertenecen al ámbito seguritario.

Se concluye que el concepto de seguridad integral, pese a ser establecido en el discurso oficial como una propuesta innovadora, no fue así, evidenciándose en otros conceptos a los que el país ya había considerado en la elaboración de instrumentos y lineamientos políticos. Pero el principal problema del concepto, viene dado por la excesiva amplitud del mismo, especialmente en lo relativo a la aplicación. Este punto resulta clave, debido a que se incorporaron instituciones que no tenían una relación directa con la perspectiva seguritaria clásica del país, mermando con ello el actuar de instituciones que tienen como objetivo fundamental, proveer seguridad al país y sus habitantes. 


\section{La necesidad de un nuevo enfoque de seguridad en Ecuador}

Al momento de establecerse el gobierno de Rafael Correa en el año 2007, uno de los elementos que marcaron los lineamientos iniciales de la mencionada administración, fue el carácter refundacional que se estableció hacia el país y su organización. En este sentido, se recalcaba la necesidad de tener un nuevo instrumento, es decir una nueva Constitución, que pudiese dar cabida a una transformación estructural del Estado, en la totalidad de sus organismos e instituciones. Y dentro de aquella lógica, el aspecto relacionado a la seguridad y los instrumentos que son participe de la misma, no quedaban atrás, lo cual quedó materializado con la adopción de la Constitución de Montecristi, del año 2008, estableciéndose de manera legal la visión "integral" de la seguridad en el país. Sin embargo, dicho concepto ya había sido establecido, desde un punto de vista discursivo por el nuevo gobierno en el año 2007, marcando una diferenciación entre una "antigua" concepción de la seguridad y un "nuevo" punto de vista sobre la misma. ${ }^{2}$ Pero el hecho de señalar un nueva concepción de la seguridad, tiene una serie de elementos que explican aquel cambio, los cuales poseen una naturaleza tanto interna como externa.

El principal elemento interno que llevó a la modificación de la concepción de seguridad del país, fue la elección del gobierno de Rafael Correa, también denominado como "Revolución Ciudadana". Este evento, el cual puede considerarse como parte de un clivaje político, tuvo implicaciones que se manifiestan en el aspecto externo, y dentro del ámbito de la seguridad. Las consecuencias internas se evidencian en dos elementos sustanciales: el nuevo gobierno, queriendo implantar un punto de vista refundacional, señaló que la perspectiva en la que el país estableció en el pasado sus prioridades en materia de seguridad, estaba basada en la doctrina de seguridad nacional. ${ }^{3}$ Esto, a juicio del nuevo gobierno, daba pie para que dicha noción doctrinaria se dejara de lado, casi de manera exclusiva por criterios ideológicos, sin tomar en cuenta las particularidades que la mencionada visión doctrinaria tenía en Ecuador. ${ }^{4}$ Pero en conjunto con señalar la necesidad de contar con un nuevo esquema que ayude al direccionamiento doctrinario de la seguridad del país, se comenzó a considerar que el mismo objeto de aplicación de la seguridad, como también de los organismos e instrumentos que son parte de la visión seguritaria ${ }^{5}$, debían modificar sus esquemas. Así, si bien la doctrina de la seguridad nacional para el caso ecuatoriano tenía como el principal elemento a resguardar al Estado en términos generales, la nueva perspectiva doctrinaria debía cambiar aquella visión, enfocándose en la sociedad que albergaba al Estado y al individuo de manera particular. Es por ello que se acogió la visión que implicaba el uso de la seguridad humana, como concepto rector

\footnotetext{
${ }^{2}$ Uno de los aspectos relevantes, es que la concepción "refundacional" también estuvo dada como consecuencia de la sucesión de gobiernos desde 2000. Por lo tanto, la visión de carencia de gobernabilidad, estaba asociada tanto a la arquitectura institucional, como a los actores que fueron participe de la misma.

${ }^{3}$ García, Bertha: "La gestión de la seguridad y defensa en el Ecuador 2007-2017, bajo un nuevo marco ideológico y geopolítico", en Correa, Loreto (comp.) (2018): Sociedad, Seguridad y Conflicto en América Latina, Ciudad de Panamá, Editorial SIEC, pp. 193-208.

${ }^{4}$ La visión de la Doctrina de Seguridad Nacional no fue algo homogéneo en Suramérica. La concepción que se tuvo en los países del Cono Sur, donde se visualizaba a un "enemigo interno", se diferenciaba con el punto de vista dado en los países andinos, donde dicha doctrina tuvo un componente mucho más desarrollista y vinculado a la industrialización. Véase al respecto Vitelli, Marina: “América del Sur: de la seguridad cooperativa a la cooperación disuasoria”, Foro Internacional, vol. 56, no 3 (julio-septiembre 2016), pp. 724-755.

${ }^{5}$ Se emplea el concepto "segurización" y sus ramificaciones, como alternativa al vocablo "securitización", como una manera de visibilizar la identidad latina en temas de seguridad, por sobre el segundo concepto, el cual posee una raíz anglosajona. Véase al respecto Rivera, Fredy (2012): La seguridad perversa: Política, democracia y derechos humanos en el Ecuador 1998-2006, Quito, FLACSO, pp. 25-66.
} 
de la seguridad durante la nueva administración, quedando aquello establecido por mandato constitucional. $^{6}$

Y un segundo elemento relacionado con el clivaje de la nueva administración en temas de seguridad, es el relacionado a la cooperación internacional que el país buscaba seguir, con el objetivo de minimizar sus amenazas y lograr un cumplimiento a sus objetivos e intereses en el ámbito exterior. Así, se tomó la decisión de disminuir la cooperación con países que no estaban ideológicamente alineados a los postulados del nuevo gobierno, teniendo como principal ejemplo la no renovación de los permisos dados a Estados Unidos para la utilización del Puesto de Operaciones Avanzadas (Forward Operating Location o FOL, por sus siglas en inglés) en la base de Manta. ${ }^{7}$ Dicha perspectiva, desde el punto de vista del nuevo gobierno, tenía como principal argumento que el territorio ecuatoriano no podía ser utilizado por una potencia extranjera, debido a que iba en contra de la visión soberana del país establecida en la nueva Constitución. Lo anterior también implicó una reducción sustancial de las actividades de cooperación en materia de seguridad con países fronterizos, especialmente con Colombia, luego del ataque que este último país efectuó a la localidad ecuatoriana de Angostura, en marzo del 2008. Sin embargo, este suceso dio como consecuencia que Ecuador estableciera una nueva arquitectura dentro del plano de la seguridad, como una forma de reforzar los esquemas conceptuales propuestos dentro del concepto de seguridad integral. ${ }^{8}$

Pese a lo mencionado, el nuevo concepto de seguridad integral también tenía implicaciones que se derivaban del contexto externo del país. Tal vez uno de los procesos con mayor influencia en la necesidad de contar con una nueva visión de seguridad para el Ecuador, fue el fin del conflicto que mantuvo con el Perú por más de 50 años, el cual se selló el año 1998. La firma de la paz con el Perú dio paso a un nuevo escenario estratégico para el país, donde la principal hipótesis de conflicto disminuía en su probabilidad de manera considerable, generando a su vez que los esfuerzos del país en la mantención de un equilibrio estratégico con su vecino del sur, fuese reemplazado por la necesidad de mantener a las Fuerzas Armadas (FF.AA.), pero con tareas diferentes. Tomando este elemento, es decir, que la posibilidad de un nuevo conflicto armado con el Perú tenía una muy baja probabilidad, es que se estableció la necesidad de utilizar el instrumento de la Defensa en otras tareas o con otros enfoques, más allá de la misión principal de los mencionados actores en torno a la preservación de la integridad

\footnotetext{
${ }^{6}$ Para una ampliación del concepto de Seguridad Humana y su aplicación al caso de América Latina, véase Paris, Roland: "Human Security. Paradigm Shift or Hot Air?", International Security, vol. 26, no 2 (otoño 2001), pp. 87102; y Fuentes, Claudia: "Seguridad Humana: Referencias Conceptuales y Enfoque Práctico para América Latina", en Rojas, Francisco (ed.) (2012): Seguridad Humana: nuevos enfoques, San José de Costa Rica, FLACSO, pp. 3354. Hay que llamar la atención de que no existe una definición que sea aceptada por todos los autores académicos y que existe una seria desviación en pocos casos al centrarse en los derechos sociales y políticos, asunto de otras disciplinas, y no en el tema central de la seguridad: La supervivencia

${ }^{7}$ La salida del FOL de Manta también tuvo repercusiones en el campo de la seguridad, ya que, pese a que se tenía una perspectiva negativa, desde el punto de vista ideológico, las labores de rastreo en torno a los flujos del narcotráfico se efectuaban de manera eficiente. Siendo así, el no contar con los instrumentos que poseía el FOL, dio como resultado un aumento en las operaciones ilegales, especialmente en la frontera norte del país. Al respecto véase Haro, Patricio: Ecuador: entre la seguridad y la inconstitucionalidad”, URVIO - Revista Latinoamericana de Estudios de Seguridad, $\mathrm{n}^{\circ} 12$ (2014), pp. 57-70.

${ }^{8}$ La Operación Fénix, acción llevada por las Fuerzas Armadas de Colombia en contra de las Fuerzas Armadas Revolucionarias de Colombia (FARC), se llevó a cabo el 1 de marzo del 2008 y afectó a un campamento del último grupo señalado, que se encontraba en la localidad ecuatoriana de Angostura, al otro lado del límite internacional. Como consecuencia de este acontecimiento, Ecuador apresuró la estructuración de su nuevo sistema de seguridad, a través de la Ley de Seguridad Pública y del Estado, publicándose en septiembre del año 2009.
} 
territorial, modificando incluso el objeto clásico de protección del Estado hacia la sociedad en su conjunto. ${ }^{9}$

Sin perjuicio de lograr una paz negociada con el Perú, quedando plasmada en un tratado internacional, y observar la evolución negativa, siempre en términos de seguridad y Defensa, que para el Ecuador estaba significando el conflicto interno colombiano ${ }^{10}$, lo cierto es que el país continuó con una concepción doctrinaria en temas de seguridad y Defensa, tomando en cuenta la noción de la amenaza convencional como el principal elemento que sustentaba la doctrina de sus fuerzas. Esto es posible de visualizar en los diferentes Libros de la Defensa Nacional, publicados en los años 2002 y 2006, donde la concepción de la disuasión y la mantención de la integridad territorial frente a las amenazas convencionales ${ }^{11}$, seguía siendo el elemento base en la construcción de la doctrina que guiaba el accionar de la Defensa del país. No obstante, ya en aquellos documentos, se mencionada que pese a mantener una perspectiva de disuasión convencional, se veía con preocupación la afectación a la seguridad del Ecuador, los acontecimientos del conflicto interno colombiano, y específicamente las consecuencias en la frontera norte del país.

Pese a lo mencionado, existen algunas posturas que señalan que la inclusión de un nuevo concepto de seguridad en el Ecuador, no solamente puede explicarse por el clivaje político interno o por la disminución de probabilidad de una amenaza convencional, particularmente con el Perú, sino que se tiene que entender desde una óptica más regional. En este sentido, se indica que desde comienzos del siglo XXI, la región suramericana vivía un período donde la democracia reinaba y, por ende, los conflictos territoriales se habían solucionado, o bien, los que quedaban pendientes se gestionaban a través de los canales que implicaba una resolución pacífica de los mismos. ${ }^{12}$ Pero lo más relevante, era el hecho de que se tenía una concepción hemisférica generalizada, posterior a los ataques del 11-S, que las principales amenazas a los países no provenían de los entes estatales, sino que de grupos criminales organizados que operaban al margen de la ley, y que utilizaban medios como el terrorismo, para el cumplimiento de sus objetivos, ya sea a nivel político u económico. Es por ello que la visión de que los Estados debían ir más allá en la configuración de sus estrategias nacionales y clásicas en los ámbitos de la seguridad y la Defensa, estableciendo una mirada "multidimensional", tanto de los

\footnotetext{
${ }^{9}$ La visión de la protección de la integridad territorial, es el principal elemento jurídico en la justificación de la mantención de los cuerpos armados institucionalizados en América del Sur. Y en el caso de Ecuador, aquello no es la excepción. Sin embargo, producto de una modificación a la Constitución de dicho país en el año 2015, se estableció un nuevo rol a las Fuerzas Armadas, en apoyo a la Seguridad Integral. No obstante, cabe señalar que dicha modificación constitucional fue revocada en agosto del 2018. Véase al respecto González, Mario: "Corte Constitucional deroga las enmiendas constitucionales aprobadas en el 2015”, El Comercio, 1 de agosto de 2018, en https://www.elcomercio.com/actualidad/corteconstitucional-analisis-enmiendas-constitucion-derogacion.html ${ }^{10}$ Véase al respecto Rivera, Fredy: "Plan Ecuador: oportunidad perdida, oportunidad posible", en Guzmán, Ivonne (ed.) (2018): El Contagio. Fin de la Isla de Paz, Quito, Mediato, pp. 87-110.

${ }^{11}$ Se entiende como "amenaza convencional", aquella que proviene de un actor estatal. Con ello, se establece la diferencia con las "amenazas no convencionales" o "no tradicionales", en las que se reconoce la existencia de otros actores que generan perjuicios a los países, pero que no son Estados como tal. Al respecto véase Cabrera, Lester: "Reflexiones en torno a los conceptos de nuevas amenazas, amenazas emergentes y amenazas no tradicionales", Escenarios Actuales, vol. 17, no 1 (2012), pp. 17-26.

${ }^{12}$ Históricamente, América del Sur ha sido considerada como una de las regiones más pacíficas del mundo, tomando en cuenta los conflictos interestatales que se han dado desde la aparición de los Estado-Nación. Pero lo anterior no necesariamente que la región goce de un estado de no violencia doméstica con alcance transnacional. Véase al respecto Kacowicz, Arie y David Mares: "Security studies and security in Latin America: the first 200 years", en Mares, David y Kacowicz, Arie (eds.) (2016): Routledge Handbook of Latin American Security, New York, Routledge, pp. 11-30.
} 
fenómenos que les afectaban, como de las soluciones que se propusieran para combatir a las amenazas. $^{13}$

Con lo mencionado, es posible indicar que el proceso que evidenciaba el país con la llegada del gobierno de la "Revolución Ciudadana" en 2007, en temáticas de seguridad y Defensa, estaba en franca evolución. Había una clara disminución de la probabilidad de un conflicto convencional, especialmente desde la firma de la paz con el Perú; pero por el contrario, la aparición de nuevas amenazas, centradas especialmente en el caso de la frontera norte, colocaba a la doctrina empleada hasta aquel momento, en un plano de abierta evaluación. Esto, sumado a la noción ideológica del nuevo gobierno, establecieron las pautas para una nueva concepción de la seguridad, denominada seguridad integral, la que tenía un fuerte componente ideológico ligado al pensamiento dominante de la nueva administración gubernamental.

\section{Seguridad Integral: conceptualización y trasfondo}

Los primeros indicios de la visión de la seguridad integral en Ecuador, es posible encontrarlos en el mismo texto de la constitución de Montecristi, particularmente en su artículo 3, numeral 8, el cual establece que es deber primordial del Estado "garantizar a sus habitantes el derecho a una cultura de paz, a la seguridad integral y a vivir en una sociedad democrática y libre de corrupción." ${ }^{14}$ Lo que llama la atención de esta primera aproximación al concepto en sí, es que se le asigne un carácter constitucional al mismo. Pero el problema en sí radica en su nula conceptualización, dentro de dicho cuerpo legal. No obstante la inexistencia de un concepto que ayude a delimitar el campo de acción de la seguridad integral, es posible establecer dos grandes lineamientos sobre el mismo: uno sobre las implicancias que tiene al estar dentro de un cuerpo legal del más alto nivel; y por otro lado, la relaciones de fondo, en términos tanto valóricos como doctrinarios, que el concepto busca generar.

Al considerar que la seguridad integral se encuentre dentro de la norma más relevante, tomando en cuenta un Estado de Derecho, establece un alto grado de cumplimiento y obligatoriedad, tanto desde el Estado mismo como de las personas que son parte de aquella institución política. Pero el caso del concepto de seguridad integral, al no tener una conceptualización explícita en el mencionado cuerpo legal, deja en el plano del "deber ser", así como en un plano de interpretación amplia de los diversos actores involucrados, las implicancias y alcances que el concepto pueda tener. Mientras que por otro lado, se puede establecer una vinculación entre el "espíritu" de la norma jurídica fundamental, y el concepto como tal. Desde aquel punto de vista, la norma se encuentra asociada a la concepción del "buen vivir", como un patrón que "direcciona" a la constitución. Dentro de dicha contextualización, la seguridad integral serviría como un concepto que integraría la noción mencionada, especialmente en lo que, eventualmente, radicaría su objetivo. Sin embargo, dicha asociación nuevamente refleja un entendimiento amplio o carente de vinculación con alguna perspectiva seguritaria efectiva, donde se busque el resguardo o protección de algún bien, objeto o proceso en particular.

Desde un punto de vista cronológico, es posible evidenciar que a comienzos del año 2008, y específicamente el 1 de marzo de dicho año, se produjo el ataque de parte de Colombia a la localidad Ecuatoriana de Angostura, con el objetivo de dar de baja a integrantes clave de las FARC. Este hecho, además de representar una clara violación a la soberanía del Ecuador, estableció la necesidad de llevar a un nuevo nivel la ejecución de la seguridad integral. Es por ello que el por entonces denominado Ministerio Coordinador de Seguridad Interna y Externa,

\footnotetext{
${ }^{13}$ Celi, Pablo: "La seguridad multifuncional en la región”, en Alda, Sonia y De Sousa, Susana (eds.) (2015): La multidimensionalidad de la seguridad nacional: retos y desafíos de la región para su implementación, Madrid, Instituto Universitario General Gutierrez Mellado, pp. 13-30.

${ }^{14}$ Asamblea Nacional del Ecuador (2008): Constitución de la República del Ecuador.
} 
elaboró el primer instrumento orientador de la seguridad en el país, bajo el contexto del nuevo gobierno, denominado "Agenda Nacional de Seguridad Interna y Externa". En dicho instrumento, más allá de señalar de manera explícita la existencia del nuevo concepto de seguridad que rige en el país, desde el advenimiento de la "Revolución Ciudadana", se otorga un argumento para la sustentación del mismo, el cual se basa en el cambio del objeto a proteger; es decir, se pasa de una noción de protección del Estado como un todo, considerado como un actor absoluto, al resguardo de un proceso que posee características sociales, como lo es el ámbito de las condiciones de vida de los ecuatorianos. Con ello se reduce la prioridad de la protección de la soberanía nacional y colocándola a la par con otros procesos. ${ }^{15} \mathrm{Y}$ pese a que la agenda no otorga una definición específica sobre lo que comprende la seguridad integral, el citado documento menciona que el concepto tiene como objetivo establecer una nueva política de seguridad que se encuentre sustentada en el respeto a los Derechos Humanos, la protección de la soberanía que poseen los pueblos del país, así como un apego a la democracia como forma de gobierno. ${ }^{16}$

Uno de los puntos que el documento plantea, es el hecho de la protección que la seguridad integral efectúa con respecto a la soberanía. Este aspecto no representaría ninguna novedad dentro del entendimiento de un proyecto seguritario; no obstante, la concepción de soberanía a la que se hace referencia, no puede comprenderse como un concepto absoluto, perspectiva que predomina a las concepciones clásicas de la seguridad ${ }^{17}$, si no que desde un punto de vista donde la protección de la soberanía recae en la sociedad en sí y en los grupos sociales que son parte de la misma. Además, y siempre de acuerdo a la citada agenda, aquella seguridad a la soberanía debe ayudar que el país pueda tener mejores relaciones con otros Estados, promoviendo el intercambio de informaciones, bienes y servicios. Por lo tanto, la soberanía tiene una perspectiva más amplia, dejando de lado las nociones más tradicionales en la región, donde prevalece la protección del territorio como parte de la soberanía y, por ende, la utilización de los medios e instrumentos de la Defensa para su resguardo. ${ }^{18} \mathrm{Y}$ en conjunto con este punto, la noción de la seguridad integral posee un fuerte componente en torno a las múltiples dimensiones que busca abordar, reconociendo que las amenazas que afectan a la soberanía de los grupos sociales, van más allá de los fenómenos y actores convencionales que buscaban gestionar los anteriores enfoques de la seguridad utilizados en el país, señalando de manera explícita la existencia de un "escenario multidimensional", para graficar el contexto de las amenazas no tradicionales. ${ }^{19}$ Sin embargo, en ninguna parte del texto se hace mención al concepto de seguridad multidimensional en sí, pese a que el país fue parte de la aprobación del mismo en los años 2002 y 2003, a través de las Declaraciones de Bridgetown y Monterrey, respectivamente, dentro del marco de la Organización de Estados Americanos (OEA).

Ligado a lo anterior, es que el documento no hace una omisión sobre la posibilidad de que el país haga frente a las amenazas convencionales, generando al respecto un vínculo que se deriva de la noción de "integralidad" de la seguridad. Por ello es la "integralidad" incluye al debate seguritario del país procesos tales la protección de los Derechos Humanos, la democracia como sistema de gobierno, la integridad del territorio, e incluso la diversidad cultural como parte de la soberanía del Ecuador, sin dejar de evidenciar la perspectiva tradicional de la

\footnotetext{
${ }^{15}$ Ministerio Coordinador de Seguridad Interna y Externa (2008): Agenda Nacional de Seguridad Interna y Externa, Quito, p. 95.

16 Ibíd.

${ }^{17}$ Véase Buzan, Barry (2009): The evolution of International Security Studies, New York, Cambridge University Press; y Orozco, Gabriel: "El concepto de la seguridad en la Teoría de las Relaciones Internacionales", Revista CIDOB d'Afers Internacionals, $\mathrm{n}^{\circ} .72$ (diciembre 2005 - enero 2006), pp. 161-180.

18 Thies, Cameron: "Traditional security: war and peace", en Mares, David y Kacowicz, Arie (eds.) (2016): Routledge Handbook of Latin American Security, New York, Routledge, pp. 113-126.

${ }^{19}$ Véase Ministerio Coordinador de Seguridad Interna y Externa, op. cit., pp. 94-95.
} 
seguridad y la Defensa en el país. Y para lograr aquella cualidad de "integralidad", en el mencionado plan gubernamental del 2008, también señala la necesidad de incorporar a dicha perspectiva a las diferentes instituciones del Estado que, de alguna $\mathrm{u}$ otra forma, son responsables de la gestión de la seguridad o de los procesos que ahora son parte de la seguridad integral, aunque solamente se visualiza como una perspectiva declarativa en términos generales, que no incluye una ruta de cómo se planea efectuar la "integralidad", tanto de los procesos mencionados como a nivel institucional.

Es así que, y en función de lo señalado, es que la Agenda Nacional de Seguridad Interna y Externa, al ser un documento donde se mencionan lineamientos y directrices, buscaba ser una alternativa a lo propuesto en los Libros Blancos de la Defensa, los cuales se gestaron a comienzos de la década del 2000, lo cual se puede comprender dentro del contexto "refundacional" que buscaba la nueva administración, dejando de lado una buena fracción de lo propuesto por los anteriores gobiernos, dentro del plano de la seguridad. Sin embargo, el problema de aquel documento, es que es meramente declarativo, mas no mandatorio ni tampoco orientador en un sentido estricto. Así, la naturaleza de la Agenda Nacional de Seguridad Interna y Externa, busca reflejar una intencionalidad política y una forma explícita en la comprensión de determinados conceptos y contextos. ${ }^{20}$ Es con la publicación de la Ley de Seguridad Pública y del Estado, donde el concepto adquiere una forma más cercana a la aplicación del mismo, especialmente en lo que respecta a la creación de órganos e instituciones que son parte de la seguridad integral. Al respecto, se señala en el artículo 1, que la mencionada ley tiene por objeto:

“[...] regular la seguridad integral del Estado democrático de derechos y justicia y todos los habitantes del Ecuador, garantizando el orden público, la convivencia, la paz y el buen vivir, en el marco de sus derechos y deberes como personas naturales y jurídicas, comunidades, pueblos, nacionalidades y colectivos, asegurando la defensa nacional, previniendo los riesgos y amenazas de todo orden, a través del Sistema de Seguridad Pública y del Estado." 21

Una interpretación de dicho apartado, considerando su propia ubicación dentro del cuerpo legal antes mencionado, indica que la cualidad de integral en torno a la seguridad, se relaciona con el hecho de vincular otros aspectos que pudiesen amenazar a la sociedad ecuatoriana. Lo mencionado queda refrendado por lo mencionado en el artículo 4, letra a), donde se menciona que "la seguridad pública será integral para todos los habitantes del Ecuador". ${ }^{22}$ Sin embargo, en el cuerpo legal se establece al mismo tiempo que en conjunto con la integralidad, la seguridad se logrará por medio de un "sistema de seguridad pública y del Estado", ampliando aún más la noción que se tenía del concepto, comparando esto con lo señalado en la Agenda Nacional de Seguridad Interna y Externa.

Derivado de un análisis del texto legal mencionado, es posible señalar 3 puntos relevantes dentro de lo que concierne a dicha ley, en relación con la seguridad integral. En primer lugar, se observa que la integralidad es posible interpretarla como un principio u orientación, más no como un medio o instrumento. Este aspecto es crucial, ya que involucraría de manera explícita, no solamente a procesos dentro del principio de la integralidad, sino que también a diversas instituciones que son generadoras o son parte de la gestión de dichos procesos, dentro del Estado. En segundo aspecto, se evidencia un fuerte componente de

\footnotetext{
${ }^{20}$ Véase al respecto Pacheco, Guillermo: “Los Libros Blancos: ¿Un paso para consolidar medidas de confianza?”, Revista Ensayos Militares, vol. 2, nº 1 (junio 2016), pp. 101-116; y González, Julián: "Libros Blancos de Defensa en Sudamérica: ¿Política exterior o política doméstica?”, Revista Brasileira de Estudos de Defensa, vol. 4, n 2 (2017), pp. 17-48.

${ }^{21}$ Asamblea Nacional del Ecuador (2009): Ley de Seguridad Pública y del Estado, artículo 1, inciso 1.

${ }^{22}$ Ibíd., art. 4, letra a).
} 
protección hacia la sociedad y sus diferentes actores. Como se señaló en su momento, la seguridad integral encuentra su base en la protección de la sociedad y sus diferentes componentes, lo que a su vez la vincula directamente con los postulados ideológicos del gobierno de Rafael Correa. Lo anterior da pie para comprender que el concepto de seguridad integral, además de considerar la orientación en torno a integrar procesos, también considera nuevos actores como parte del ejercicio seguritario. En otras palabras, y de acuerdo al texto legal, se añaden y consideran nuevos actores como objeto de resguardo y protección, dejando a un lado si es que dichos actores se encuentran dentro de la lógica seguritaria de las instituciones que son responsables de la seguridad. Y en tercer lugar, se destaca en la totalidad de la ley, que nuevamente se carece de una definición explícita y tácita de lo que es la seguridad integral, pese a que en el mismo cuerpo legal se otorgan conceptos orientadores para el funcionamiento de nuevas instituciones, como es el caso de la Secretaria Nacional de Inteligencia (SENAIN). ${ }^{23} \mathrm{El}$ problema de lo anterior, radica en que al no tener una clara conceptualización de lo que se entiende por seguridad integral, el actuar de los nuevos organismos, como lo fue la SENAIN, otorgaba una amplia interpretación sobre el actuar de dichos organismos, en base a que estaban cumpliendo con un precepto como la seguridad integral, generando que su actuar fuera constantemente criticado, al no cumplir con los pocos y amplios preceptos sobre los cuales fue creada dicho organismo. ${ }^{24}$

Pese a que el concepto de seguridad integral viene siendo señalado como el aspecto clave dentro del cambio de noción seguritaria en el Ecuador, es solamente a partir del año 2011, con la publicación del primer Plan Nacional de Seguridad Integral 2011-2013, donde se pueden observar definiciones explícitas de lo que comprende la seguridad integral. Es así como el mencionado plan refuerza la idea de que la seguridad integral posee dicha categorización debido a la cualidad de "integrar" diferentes elementos dentro del concepto mismo de seguridad. Al respecto, el plan establece que:

"El enfoque integral hace referencia al sentido de un Sistema Integrado de Seguridad, que abarca todos los ámbitos del ser humano y del Estado, sin dejar nada al azar. Tiene que ver con la integración de todas las esferas de la seguridad, las mismas que se complementan y se integran en un solo sistema, el Sistema de Seguridad Publica y del Estado. Allí encontramos la seguridad internacional, la seguridad interna, la Defensa Nacional, la seguridad económica, la soberanía alimentaria, la seguridad ambiental, entre otras." 25

En la mencionada cita, se señala que la seguridad integral es una condición, que busca unir una serie de "otras seguridades" dentro de un mismo concepto. Es decir, la seguridad integral abarca otro tipo de enfoques en la seguridad, como lo son la seguridad interna, internacional, la Defensa, la seguridad económica, ambiental y también la seguridad pública y ciudadana. Con ello, el concepto adquiere una mayor amplitud en torno a lo que busca abarcar. Y como una forma de complementar la anterior cita, en el Plan se señala que:

"La seguridad con Enfoque Integral, es la condición que tiene por finalidad garantizar y proteger los derechos humanos y las libertades de ecuatorianas y ecuatorianos, la gobernabilidad, la aplicación de la justicia, el ejercicio de la democracia, la

\footnotetext{
${ }^{23}$ Como se señaló, la propia Ley de Seguridad Pública y del Estado, establece conceptos que permiten una orientación en las funciones de las nuevas instituciones que el cuerpo legal crea. Un ejemplo claro de aquello es lo que se evidencia con el concepto de inteligencia, el cual es expresamente definido en el artículo 14, letra a) de la mencionada ley.

${ }^{24}$ Rivera, Fredy: "Inteligencia estratégica e inteligencia política: los claro-oscuros del caso ecuatoriano" (2016): Inteligencia estratégica contemporánea, Sangolquí, ESPE, pp. 133-148.

${ }^{25}$ Ministerio Coordinador de Seguridad (2011): Plan Nacional de Seguridad Integral 2011-2013, p. 14.
} 
solidaridad, la reducción de las vulnerabilidades, la prevención, protección, respuesta y remediación ante riesgos y amenazas." 26

Uno de los puntos que llaman la atención, además de la excesiva amplitud que recoge la condición de integralidad de la seguridad para el Ecuador, es el hecho de que se mezclan aspectos que no necesariamente tienen una condición seguritaria, dentro de la protección que debe brindar la seguridad integral. E incluso como forma de complementar lo anterior, está el hecho de que se colocan en un nivel similar procesos tales como la Defensa y la seguridad económica y ambiental. Este aspecto resulta relevante, tomando en cuenta que dicha perspectiva significa o rebajar el actuar de los clásicos cuerpos e instituciones que brindan seguridad y/o Defensa al país, como lo son la Policía Nacional o las FF.AA., a labores a las que no se encuentran prepararas. $\mathrm{O}$ en su defecto, colocan al mismo o similar nivel decisional, siempre dentro del plano de la seguridad, a instituciones que no poseen cualidades o una tradición en la administración de temas de seguridad propiamente tal, como es el caso del Ministerio de Justicia o la denominada Secretaria de Gestión de Riesgos. Y pese a la inclusión de las instituciones mencionadas, entre otras, aún no queda claro el aporte específico de las mismas al concepto, de manera explícita. En otras palabras, la perspectiva de la seguridad integral sigue observándose como meramente orientadora, no estableciendo una claridad en torno a las acciones que las diferentes organizaciones y actores deberían efectuar al actuar bajo el concepto en sí.

Finalmente, es el año 2014 donde se publica el último de los Planes Nacionales de Seguridad Integral, con una vigencia desde el 2014 al 2017, coincidiendo este último año con las elecciones para el cargo de Presidente de la República. Y a diferencia de los anteriores documentos, incluyendo la Agenda Nacional de Seguridad Interna y Externa, este nuevo plan se destaca por su extensión y profundidad en lo que concierne a la justificación y vinculación teórica del concepto de seguridad integral. Pero además, este documento busca establecer parámetros para que el concepto vaya más allá de la orientación o condición sobre la integralidad de la seguridad, generando eventuales bases para la adopción de una política pública encaminada a la construcción de elementos explícitos en torno a la seguridad integral. Para ello se incluyen dentro del plan, una serie de cifras e indicadores que van de la mano con los objetivos que se plantearon en torno a la seguridad integral, como es la reducción de la pobreza, la desigualdad, e incluso variables que buscan expresar mejorar en aspectos educacionales a nivel general. Y desde un punto de vista ideológico, este último plan también enfatiza, a diferencia de los anteriores, la necesidad de haber contado con un nuevo concepto de seguridad en el país, tomando como referencia la perspectiva de la doctrina de la seguridad nacional, asociándola a un instrumento conceptual de injerencia de terceros países violaciones a los derechos humanos. ${ }^{27} \mathrm{El}$ mismo plan señala de forma explícita que:

"El Plan Nacional de Seguridad Integral 2014-2017 presenta importantes rupturas en relación a los instrumentos de planificación anteriores tanto en la metodología del proceso como en la formulación misma del Plan. El aspecto más relevante quizás es que se amplía el concepto de Seguridad Integral al volverlo una política del Estado operativizada por todas sus funciones en conjunto [...]."28

Lo anterior se entiende desde la perspectiva de planificación que menciona el plan. Así, para la obtención de una seguridad integral que pueda ser operativizada, el plan señala que existen tres niveles de planificación. En primer lugar, se observa un nivel que se deriva de los instrumentos rectores, como lo es el Plan en sí. En un segundo nivel, se contempla la planificación dada por

\footnotetext{
${ }^{26}$ Ibídem.

${ }^{27}$ Ministerio Coordinador de Seguridad (2014): Plan Nacional de Seguridad Integral 2014-2017

${ }^{28}$ Ibíd., p. 16.
} 
los entes que tienen por misión la vinculación entre los lineamientos macro, con la ejecución a nivel de política pública. Entre esos entes, se pueden señalar los enlaces dados por los ministerios coordinadores, como es el caso del Ministerio Coordinador de Seguridad. Y en un tercer nivel más pequeño, se encuentran las diferentes carteras ministeriales y secretarias que se encuentran dentro de la perspectiva que incluye a la seguridad integral. ${ }^{29}$

Otro de los aspectos que incorpora este último plan, siempre en relación a la construcción del concepto de seguridad integral, es la justificación teórica que se le da al término en sí. Incluso, por primera vez, se evidencia que el concepto adquiere una cualidad nacional, que pretende diferenciarse de otros conceptos similares o que guardan una relación con el mismo, como sucede con la seguridad multidimensional. Siendo así, el plan busca generar un abordaje al concepto de seguridad integral, desde la noción del "buen vivir", tomando en cuenta que dicha perspectiva configura un razonamiento que va más allá de lo estadocéntrico, incluyendo una lógica derivada de un pensamiento antropocéntrico y biocéntrico (la naturaleza). Y es finalmente este último componente, lo que diferencia y une al concepto de seguridad integral con una óptica propia de la ideología establecida por el gobierno de aquel entonces. ${ }^{30}$

Sin perjuicio de los esfuerzos que se evidencian en el último plan 2014-2017, en ayudar en la gestión del concepto de seguridad integral, lo cierto es que lo anterior puede considerarse como un esfuerzo tardío y que, incluso, aborda temáticas que complejizan aún más su grado de operativización. En este sentido, el hecho de considerar que la seguridad integral tiene una naturaleza que se extrae de la noción del "buen vivir", lo que a su vez vincula al concepto con lo biocéntrico, añade un grado de complejidad mayor a los alcances y formas de aplicar la seguridad integral en el país, debido principalmente a que no se tiene una categorización clara en cuanto a la forma de aplicar lo señalado al ámbito de la naturaleza. Pero lo que genera un mayor problema, es que se acentúa la inclusión de temas que son fundamentalmente ligados a las condiciones de desarrollo de un país, como lo es la extrema pobreza, la desigualdad o incluso los niveles de educación, a un espectro seguritario. Lo anterior, sumado a que la implementación de la política publica de seguridad, posee niveles que le dan a determinados ministerios, potestad para efectuar cambios e incluso interpretación de la política de seguridad, pero que no tienen un componente seguritario, otorga como consecuencia directa una mayor complejidad al momento de evidenciar los alcances y límites de la seguridad integral para el país.

Más allá de sistematizar como se ha venido dando el concepto de seguridad integral, observando la evolución misma del concepto, ya sea a través de instrumentos jurídicos o de documentos orientadores, cabe señalar que el concepto fue modificándose de acuerdo a las propias circunstancias políticas que evidenciaba el Ecuador, como también al hecho de que puede considerarse como un reflejo mismo de la institucionalidad del país por aquellos años. En este sentido, el concepto no fue parte de un proceso debidamente planificado, sino que hubo una serie de acontecimientos que forzaron a la modificación del mismo, e incluso a la necesidad de incorporar nuevos actores a la seguridad integral, tomando en cuenta la evolución de los acontecimientos políticos. Lo anterior se ve evidenciado en la imposibilidad de otorgar una definición que ayudara a la construcción de los eventuales procesos que se mencionaban como parte de la orientación de la seguridad integral, desde un principio, viéndose influido el mismo por sucesos coyunturales que empujaron la modificación constante de la seguridad integral, ${ }^{31}$

\footnotetext{
${ }^{29}$ Ibíd., p. 17.

${ }^{30}$ Ibíd., p. 25.

${ }^{31}$ La evolución política del Ecuador durante la administración de Rafael Correa, no estuvo exenta de problemas de índole institucional, tanto a nivel interno como externo. Y pese a que el suceso de "Angostura" el año 2008, marcó un punto de inflexión en la seguridad del país, evidenciándose en la generación de la Ley de Seguridad
} 
lo que habla de una carencia de planificación en el mediano y largo plazo, para una adecuada implementación de lo propuesto.

\section{Implicaciones en el uso del nuevo concepto en las FF.AA.}

Al momento de considerar las implicancias y consecuencias que el concepto de seguridad integral trajo al Ecuador, es también necesario tomar en cuenta un elemento no menor, el que se atribuye al momento ideológico que estaba pasando el proyecto del gobierno de Rafael Correa, tanto de alineamiento hacia otras gestiones con un pensamiento similar, así como también el consecuencia alejamiento de instituciones que representaban un eventual "dominio" de parte de Estados Unidos, al tiempo que reflejaban una perspectiva del pasado político que el nuevo gobierno deseaba dejar atrás. Con respecto al primer punto, la administración de Correa estableció un vínculo, tanto a nivel discursivo como de acercamiento político, con los gobiernos que se caracterizaban por tener una tendencia hacia el "progresismo" o ser parte del denominado "socialismo del siglo XXI". ${ }^{32}$ En este plano, dicha corriente de pensamiento establecía elementos o bases para planificar aspectos económicos y de desarrollo, donde el énfasis estaría en la perspectiva de bienestar de los pueblos, por sobre las ganancias y utilidades que pudiesen obtener las empresas privadas. Sin embargo, se agregaba el hecho de marcar un punto de diferencia con anteriores administraciones, especialmente desde la perspectiva del discurso en la política exterior, donde se establecía una distancia con Estados Unidos y las instituciones y organismos internacionales, especialmente los ligados a instancias de cooperación y financiamiento, donde la imagen de aquel país era considerada como "hegemónica".

Como consecuencia de la línea de pensamiento mencionada, es que la adquisición del concepto de seguridad integral posee una composición ideológica, dada tanto por los elementos que son parte misma del término, como también por el contexto internacional en que América Latina en general, y Ecuador en particular, se vio envuelto por aquellos años. Es por eso que pese a que algunos preceptos de la seguridad integral encuentran un eco en la perspectiva de la seguridad multidimensional, en ningún documento que implique un desarrollo de lo que es la seguridad integral se hace referencia alguna del concepto aceptado por el país en los años 2002 y 2003, al haberse establecido dentro del marco de diversas reuniones de la OEA. Esto último se explica por la perspectiva ideológica del nuevo concepto, como también por la línea de política exterior, seguridad y Defensa durante la administración de Rafael Correa. Lo anterior además, se vio reflejado en acontecimientos tales como la no renovación del convenio del FOL del Manta, y el ataque a Angostura por parte de Colombia, exacerbando en este último caso, el discurso anti-imperialista del gobierno de turno.

En conjunto con lo señalado, al tener la seguridad integral una vinculación con proyectos políticos e ideológicos definidos, los cuales buscaban plasmar un sentido "refundacional" para el Estado, los actores involucrados en la planificación y ejecución de concepto, especialmente en lo que respecta a su doctrina, es decir las FF.AA. y la Policía Nacional, tuvieron un papel no menor dentro de la aplicación de los preceptos que involucraba la seguridad integral, especialmente en los primeros actores mencionados. Históricamente, las

Pública y del Estado en el 2009, la sublevación de la Policía Nacional el 30 de septiembre del 2010, también puede considerarse como un punto de quiebre en la gestión de la seguridad, e incluso, para algunos organismos internacionales como la Unión de Naciones Suramericanas (UNASUR), fue un intento de desestabilización del gobierno. Estos acontecimientos también pueden ser incorporados como factores de cambio tanto en la necesidad de institucionalizar el concepto de seguridad integral, como también en una eventual modificación o adaptación del mismo, a las nuevas circunstancias del país. Un caso de como una de las perspectivas que era parte de la seguridad integral, la inteligencia, fue modificándose de acuerdo a las "coyunturas críticas", puede evidenciarse en Piedra, Lorena (2012): Coyunturas críticas en los sistemas de inteligencia, Quito, FLACSO-Ecuador.

${ }^{32}$ Zepeda, Beatriz: "La Política Exterior del Ecuador Durante el Gobierno de Rafael Correa: Un Balance", en Mathieu, Hans y Niño, Catalina (eds.) (2011): Anuario 2011 de la Seguridad Regional en América Latina y El Caribe, Quito, Friedrich Ebert Stiftung, pp. 114-126. 
FF.AA. de América del Sur en general, y las ecuatorianas en particular, han tenido una concepción de protección y resguardo hacia el territorio, dentro de una perspectiva soberana. Incluso, dentro de un punto de vista histórico e institucional, los cuerpos armados han sido catalogados en determinados momentos, como los actores llamados a garantizar el "orden institucional" e, incluso el sistema democrático. ${ }^{33}$ Pero más allá de las características y responsabilidades dadas a los cuerpos armados en un período histórico particular, dentro del plano doctrinario, las FF.AA. han tenido una cualidad de actuar, principalmente, bajo la noción de la disuasión y específicamente hacia actores denominados convencionales; es decir, otros ejércitos regulares que responden a los objetivos de otros Estados.

Producto de lo anterior, es que la respuesta de las FF.AA. ecuatorianas en su mayoría, en lo relativo a la aplicación de los preceptos del concepto de seguridad integral, puede catalogarse como dual. Si bien es cierto que por un lado se comenzaron a adoptar medidas para incorporar las nociones que implicaba el concepto de seguridad integral, especialmente en lo referente al actuar de las fuerzas en torno a las "nuevas amenazas" y en el posicionamiento de las mismas en tareas o misiones que fueron siendo conocidas como subsidiarias ${ }^{34}$, fue el componente político lo que mayormente forzó a las FF.AA. a considerar nuevos enfoques dentro de su aparato doctrinario. Un ejemplo de ello fue que, derivado del direccionamiento político que el gobierno estableció hacia los componentes armados, se planteó la necesidad de disminuir las brechas y atenuar las diferencias entre los oficiales y los miembros del cuadro permanente, ya sea a través de medidas que iban desde la supresión de los comedores diferenciados, hasta la aplicación de una nueva medición para el cálculo de las pensiones, tomando como uno de los aspectos rectores la disminución de la jubilación entre los escalafones mencionados. ${ }^{35}$

Este tipo de medidas, especialmente las que derivaban del estamento político en torno a un cambio en la forma de actuar de las FF.AA. ecuatorianas, especialmente en aquellas áreas en donde se relacionaban en la construcción e implementación de preceptos doctrinarios, comenzaron a generar diferencias entre miembros de las FF.AA. y funcionarios de gobierno de alto nivel, así como problemas dentro de las mismas FF.AA. ${ }^{36}$ Pero el hecho más relevante, radica en la pérdida de las capacidades operativas de las FF.AA., debido a que la implementación de un cambio de doctrina, donde se establezca la necesidad de un nuevo enfoque en lo relativo al objeto a proteger, no es posible incorporarlo en un corto plazo. Por lo tanto, las FF.AA. se vieron inmersas en un proceso de cambio no planificado, tanto a nivel doctrinario como en sus mismas capacidades operativas, lo que dio como directa consecuencia una abierta crítica a los postulados gubernamentales y, por ende, una pérdida de los medios empleados para hacer frente a las amenazas que se ciernen sobre el país.

\footnotetext{
${ }^{33}$ Al respecto véase Stepan, Alfred (1988): Rethinking Military Politics: Brazil and the Southern Cone, Princeton, Princeton University Press; y Haro, Patricio (2017): Fuerzas armadas, pretorianismo y calidad de la democracia: Ecuador y Uruguay, Quito, FLACSO Ecuador.

${ }^{34}$ Pérez, Diego y Cruz, Galo (2015): Transiciones para la conducción civil y reformas en el sector defensa en Ecuador, Quito, FES-ILDIS.

${ }^{35}$ Redacción Política: "Ricardo Patiño reitera que se busca justicia en pago de pensiones militares". El Universo, 18 de julio de 2016, en https://www.eluniverso.com/noticias/2016/07/18/nota/5698200/ricardo-patino-reitera-queno-se-incrementaran-pensiones-que-esten

${ }^{36}$ Un ejemplo de lo anterior se visualiza con la iniciativa del gobierno, de trasladar a miembros de las FF.AA., a otros rubros de seguridad, pero que no se vinculan, directamente, con las funciones tradicionales ni a la doctrina por las que fueron formados, lo que dio como resultado una abierta pugna dentro de las FF.AA. por un lado, y entre las FF.AA. y el gobierno por otro. Al respecto, véase Redacción Ecuavisa: "Militares se unirán a nuevos grupos de seguridad". Ecuavisa, 2 de diciembre de 2013, en https://www.ecuavisa.com/articulo/noticias/actualidad/47650-militares-se-unirian-nuevos-grupos-seguridad
} 
No obstante, y pese a que posteriormente las misiones subsidiarias tuvieron un reconocimiento constitucional a partir del año 2015, uno de los componentes en los que históricamente las FF.AA. ecuatorianas han tenido una fuerte presencia, es en diferentes acciones y labores tendientes a aumentar los niveles de desarrollo y bienestar de la sociedad, e incluso otorgar un grado de protección a actividades que son consideradas como "estratégicas" para el país. Un ejemplo de lo anterior se visualiza en el grado de responsabilidad que tienen los cuerpos armados en la protección de la infraestructura y logística asociada a la extracción y traslado del petróleo. Por ende, lo calificado como "subsidiario", no puede considerarse como algo novedoso o logrado producto de la perspectiva integral de la seguridad, sino como un reconocimiento y sistematización de actividades que las FF.AA. venían ejecutando desde hace varios años, pese a que el gobierno de turno de por aquel entonces, no compartía aquella visión.

Y si bien el primer componente de la perspectiva dual indicaría que las FF.AA. se movieron hacia una noción donde las amenazas tradicionales quedaban de lado, aquello no es así. Es por eso que el segundo componente de dicha perspectiva dual, se vincula al hecho de que si bien por un lado las FF.AA. consideraron las nociones propuestas en torno a la seguridad integral, dichas consideraciones no fueron totales ni tampoco tuvieron un grado efectivo en lo que respecta al cambio doctrinario de las fuerzas. Esto, producto de que, siempre en el plano doctrinario, no se abandonó la visión tradicional de amenaza, tomando en cuenta que pese a que el equilibrio estratégico entre Ecuador y sus países vecinos se inclinaba a estos últimos, se contemplaba una concepción de protección al Estado en términos territoriales. Esto se comprueba tanto a través de la preparación operacional de los medios bélicos, como también en los diferentes encuentros en los que los propios oficiales manifestaban la necesidad de aumentar la capacidad de los medios tradicionales con los que contaban las FF.AA. ${ }^{37}$

Siendo así, una de las principales implicancias negativas del concepto de seguridad integral dentro de los componentes armados del país, es que si bien intentó establecer una nueva perspectiva doctrinaria, la amplitud y vaguedad del mismo imposibilitó aquello. En este plano, si bien es cierto que se señalaba la necesidad de tener un enfoque más amplio de la seguridad y la Defensa en el país, tomando en cuenta las nociones más contemporáneas en el campo de los estudios estratégicos, lo cierto es que la visión doctrinaria de las FF.AA. no es posible modificarla sin establecer una claridad en lo nuevo y, especialmente, en lo que se desea proteger y cómo se desea resguardar, especialmente si se posee una noción donde es la disuasión lo que determina las capacidades y la formación de los cuadros. ${ }^{38}$ Lo anterior se asocia con la propia naturaleza de los miembros de los cuerpos armados y su formación, tomando en cuenta que la incorporación de otras misiones, más allá de las fundamentales, implica una preparación diferente, situación que no se generó tanto por la carencia de perspectiva de parte de las instituciones encargadas de la planificación del concepto, como también por la resistencia de los mismos actores responsables de su ejecución.

Otra de las consecuencias negativas que se pudieron visualizar al momento de buscar la aplicación y ejecución en la práctica del concepto de seguridad integral, fue la separación que se produjo entre "civiles" y "militares", como parte de aquella diferenciación entre los actores que establecían la planificación del concepto, desde un punto de vista político, y aquellos encargados de su posterior ejecución. Aquella separación, que es propia para comprender las

\footnotetext{
${ }^{37}$ En este punto, las FF.AA. ecuatorianas sostuvieron una abierta crítica al manejo de la Defensa por parte de una buena parte de los ministros de la cartera, debido a que no se les proporcionaba los medios necesarios para poder proteger la integridad territorial del país. Y pese a que hubo un aumento en el gasto de la Defensa durante la administración de Rafael Correa, el énfasis estuvo en el gasto corriente, es decir, salarios. Véase al respecto Redacción Plan V: "El legado del correato en armamento y gasto militar". Plan V, 3 de septiembre de 2018, en http://www.planv.com.ec/historias/sociedad/el-legado-del-correato-armamento-y-gasto-militar

${ }^{38}$ Morgan, Patrick (2003): Deterrence Now, Cambridge, Cambridge University Press.
} 
relaciones entre los actores que poseen el monopolio de las armas y los que detentan el poder político dentro del campo de la ciencia política, se debe articular sobre bases tanto políticas como culturales de la realidad que se tenga en consideración. Y pese a que el país se encuentra dentro del contexto suramericano y andino para poder analizar el comportamiento y evolución de las relaciones civiles-militares en un conjunto ${ }^{39}$, la realidad indica que el país evidenció una interpretación compleja de lo que implica la concepción de la división antes señalada. En este sentido, la comprensión de lo que se generó en el ámbito de las relaciones civiles-militares durante el gobierno de Rafael Correa, estuvo imbuido tanto por el contexto refundacional señalado en su momento, como también por dos aspectos relevantes: la incorporación del concepto de seguridad integral como un marco de entendimiento entre los diferentes componentes de la Defensa; y una perspectiva ideológica que evidenciaba a las FF.AA como actores que si bien cumplían un papel relevante en el ámbito de la Defensa, no eran los únicos, por lo que se tendió a buscar una "desmilitarización" de la Defensa, dando como consecuencia una fuerte resistencia en la implementación del concepto, por parte de las FF.AA. ${ }^{40}$

El problema además, radicó en el hecho de que el gobierno de Rafael Correa, no buscó una negociación sobre las competencias en torno a la función militar, sino que una imposición de términos dentro del plano doctrinario, logrando con ello una clara intervención de la esfera política en el contexto de las FF.AA. Pero el tema fue más allá, debido a que las implicancias de ejecutar y aplicar el concepto de seguridad integral, evidenció la carencia de formación, de parte del espectro político, en temas relativos a la cultura e institucionalidad militar. Dicho de otro modo, no se tenía una noción ni conocimiento de alguno de los preceptos básicos que son parte de las relaciones civiles-militares, tanto desde un punto de vista amplio como específico, enfocado en la realidad de América Latina y Ecuador. ${ }^{41}$ Es por ello que la seguridad integral no se gestionó como tal, sino que fue en una buena parte, una imposición de parte del poder político hacia los componentes armados, justificando su actuar sobre una base conceptual y teórica que se evidencia en los mismos planes de seguridad. Pero como se señaló en su momento, los argumentos académicos o teóricos, fueron en realidad utilizados para complementar la justificación ideológica en la creación y posterior implementación del concepto.

Es por eso que la seguridad integral, al ser un concepto que buscaba reorientar las misiones de los cuerpos encargados de la seguridad y la Defensa en el país, también permitía establecer canales de diálogo y comunicación entre los actores responsables de los sectores mencionados. Pero la comprensión de lo que implica dicha relación no fue adecuadamente comprendida, debido a que se configuró una relación de subordinación desde el ámbito "civil" hacia el sector militar, siempre considerando el hecho de que los militares no son el único elemento de la Defensa. Aquella perspectiva, si bien encuentra un eco con las perspectivas más contemporáneas en la comprensión de la seguridad y la Defensa ${ }^{42}$, posee a su vez un fuerte componente ideológico, considerando que los militares no detentan el monopolio de la función Defensa. Y las acciones que evidencian lo anterior en el Ecuador, van desde una noción de una inclusión minoritaria de oficiales de las FF.AA., en planificaciones académicas relativas a seminarios o cursos de especialización, hasta el cambio de las propias funciones de los militares,

\footnotetext{
${ }^{39}$ Nunn, Frederick (2011): Relaciones Militares Civiles Sudamericanas en el Siglo XXI, Santiago, Academia de Guerra del Ejército de Chile.

${ }^{40}$ Pérez, Diego: "Fuerzas Armadas en Ecuador. Ideología, políticas públicas y diálogo civil militar", en Namihas, Sandra (edit.) (2018): El Nuevo rol de las Fuerzas Armadas en Bolivia, Brasil, Chile, Colombia, Ecuador y Perú. Red de Política de Seguridad, Lima, Pontificia Universidad Católica del Perú - Konrad Adenauer Stiftung, pp. 101-138.

${ }^{41}$ Pérez, Diego (2018): Liderazgo político y transformaciones institucionales: relaciones Ejecutivo-partidos políticos-Fuerzas Armadas en Ecuador (1996-2006), Quito, Instituto de Altos Estudios Nacionales.

${ }^{42}$ Véase Saint-Pierre, Hector: "'Defense' or 'Security'?: reflections on concepts and ideologies", Contexto Internacional, vol. 33, n 2 (2011), pp. 407-433.
} 
siendo este último punto, en conjunto con el tema de la rebaja de las pensiones en la oficialidad, lo que más problemas provocó entre la dirección política de la Defensa y los cuerpos armados. ${ }^{43}$ En otras palabras, si bien la seguridad integral indicaba que habían más actores responsables de la dirección y gestión de la seguridad y Defensa del país, no se estableció una priorización en cuanto a las instituciones que eran responsables, en términos de medios para efectuar la labor encomendada, para la aplicación de la seguridad integral. Aquella situación, sumado al hecho de que de acuerdo a lo establecido en los mismos planes de seguridad integral, y específicamente el del año 2014-2017, donde se sugería la necesidad de gestionar la implementación de la seguridad integral a través de 3 niveles diferentes, dentro del discurso, daba potestad a diferentes instituciones para planificar su perspectiva en torno a la aplicación de la seguridad integral, lo que restaba relevancia o provocaba diferencias, entre las mismas instituciones u organismos que eran parte del proceso de seguridad para el país.

Otra de las repercusiones que se evidencian en el concepto, es que derivado de su amplitud y su vaguedad, no se tuvo una real conciencia de cómo se debía ejecutar, en términos efectivos y de política pública. Esto último no es un aspecto menor, debido a que, si bien se incorporaron nuevos actores al proceso seguritario, aquello no necesariamente dio como resultado una mejora de los niveles de seguridad en el país o, incluso, provocó problemas en torno a las funciones que los actores debían efectuar. Uno de los principales ejemplos al respecto fue lo relativo a la inteligencia. Producto de la necesidad de contar con una institución que brindara información procesada y con valor estratégico al Presidente de la Republica, con el objetivo de tomar mejores decisiones, se creó la ya mencionada SENAIN, al tiempo que se reorganizó la totalidad del sistema de inteligencia nacional. Y si bien el objetivo primario era la generación de insumos en el plano estratégico para el país, su funcionamiento tuvo un fuerte énfasis en relación a acciones de índole político, además de reducir las potestades de la inteligencia militar. ${ }^{44}$

\section{Conclusiones}

La interpretación que se pudiese efectuar del concepto de seguridad integral, no solamente reviste una complejidad en lo que se refiere a su comprensión teórica, sino que también da paso para que sea un concepto asociado a un determinado período en la historia política del Ecuador. Estos aspectos a su vez le otorgan una fuerte carga ideológica al concepto, lo que no da pie para un análisis exhaustivo y objetivo en torno al mismo. Sin embargo, y más allá de los elementos y contextos que rodean a la concepción de la seguridad integral, es posible señalar que Ecuador abrió la discusión a nuevas temáticas, pero desde un punto de vista erróneo en la conceptualización, procesos y aplicaciones institucionales, dentro de la lógica de la seguridad integral. Y se menciona erróneo, tanto por el contexto político y estratégico sobre el cual se buscó implementar, la noción refundacional de la seguridad que se buscaba, y la perspectiva de un concepto complejo de teorizar y aplicar.

El contexto político, tanto a nivel interno como externo, no era el adecuado para avanzar en la construcción de un nuevo concepto de seguridad, especialmente uno que implicara una fuerte desvinculación de los preceptos más clásicos y tradicionales de la seguridad nacional.

\footnotetext{
${ }^{43}$ Además de las diversas manifestaciones que sufrió el entonces Ministro de Defensa Ricardo Patiño en contra de los cambios propuestos por el gobierno al sistema de pensiones de las FF.AA., es posible destacar la reacción de los miembros del Comando Conjunto de las FF.AA. Ecuatorianas, a fines del año 2013, donde manifestaron abiertamente en un video, su disconformidad con los cambios de funciones hacia las FF.AA. Al respecto véase Redacción Política: "En video, Alto Mando pide a soldados evitar rumores sobre recorte en FF.AA.”, El Universo, 12 de diciembre de 2013, en https:/www.eluniverso.com/noticias/2013/12/12/nota/1908226/video-alto-mandopide-soldados-evitar-rumores-sobre-recorte-ffaa

${ }^{44}$ Véase al respecto Ordóñez, María Dolores y Cruz, Galo: "La inteligencia militar ecuatoriana en la sociedad del riesgo", URVIO - Revista Latinoamericana de Estudios de Seguridad, no 21 (2017), pp. 56-69.
} 
Aquello se deriva de que el país se encontraba en pleno proceso de cambio de institucionalidad, pero los cambios en un aspecto doctrinario, no se puede generar en el corto plazo, debido a que la puesta en marcha de los nuevos preceptos, así como las lecciones aprendidas, requieren de un tiempo considerable. Pero además, no había una claridad en torno a lo que realmente se buscaba con el concepto de seguridad integral, lo que añadía una mayor dificultad a la hora de su implementación. Y en segundo lugar, el contexto externo, siempre desde un punto de vista seguritario, no era el óptimo, debido a las consecuencias que ya se evidenciaban del conflicto interno colombiano, en la seguridad del Ecuador. Aquello queda reflejado con la poca capacidad de acción que tuvo el Estado ecuatoriano, al momento de evitar que se produjese el ataque de la localidad de Angostura, y las consecuencias negativas que el mismo conflicto interno de Colombia, comenzó a generar especialmente en su frontera norte.

La visión de la seguridad integral careció de una adecuada teorización y discusión, especialmente en términos de sus implicancias como concepto, y la relación que tenía su eventual implementación con los actores encargados del ejercicio seguritario. Un ejemplo de lo anterior radica en que pese a que hubo 2 planes de seguridad integral y un plan previo donde se mencionaba el concepto, ya por el año 2007, nunca hubo una claridad en lo que representaba el concepto en sí ni tampoco en lo que tenía que considerar para su adecuada implementación. Incluso, analizando los diferentes documentos que establecieron lineamientos sobre lo que era e implicaba la seguridad integral, se visualiza una amplitud y vaguedad que no permite una adecuada comprensión del mismo, llegando incluso a complejizar el entendimiento antes de facilitarlo.

La complejidad o carencia de una explicación simple sobre el concepto, tiene una raíz que se evidencia tanto en la base epistémica de la seguridad integral, como en el hecho de desconocer factores que implicaban un cambio dentro de la doctrina de seguridad de un país como el Ecuador. El primer aspecto, es decir el origen epistémico de la seguridad integral, el cual se vincularía a la noción crítica de los estudios estratégicos, pasa por un reconocimiento de la dificultad de comprender, de manera específica, el objeto de estudio de dicha corriente. Pero el problema no radica en que se aplique una perspectiva crítica para la construcción de un concepto, sino que las bases para establecer y oficializar el nuevo concepto, provenían de una base ideológica, mas no teórica. Por lo tanto, la crítica para cimentar el concepto de seguridad integral, se transformó en una crítica en sí misma, siempre desde un punto de vista ideológico. Así, cualquier intento de debatir sobre la funcionalidad y aplicación del nuevo concepto, desde la óptica académica, se construía como una arquitectura que ayudaría a justificar la necesidad del concepto, pero siempre desde una lógica ideológica, mas no desde un punto desde las necesidades reales del país, para actualizar su arquitectura de seguridad y Defensa.

Pero tal vez el principal problema de la forma de la seguridad integral, pasa por el desconocimiento de las propias capacidades del país para afrontar los desafíos y retos que el concepto abría. La seguridad integral dio la base para ayudar a una desmilitarización de una serie de funciones relacionadas a la seguridad, pero lo cierto es que el país no tenía -ni tieneuna masa crítica que permita implementar una noción de seguridad más amplia a la convencional. Incluso, dentro del plano de los estudios estratégicos, y específicamente en lo que respecta a la Defensa Nacional, Ecuador no poseía los medios humanos, tanto de naturaleza militar como civil, para una eficiente y eficaz gestión y planificación de las diferentes políticas públicas que lleva consigo la implementación de un concepto tan amplio. Aquella cualidad fue uno de los puntos más bajos, en términos de ejecución, de la nueva visión de seguridad que estableció el gobierno de Rafael Correa. Este es, tal vez, uno de los principales que desafíos que aún enfrenta el país: la formación de una comunidad de estudios que aborde, con altura de miras y con un adecuado diagnóstico de la realidad y los medios que evidencia el Ecuador, en materia de seguridad y Defensa. 


\section{Bibliografía}

Asamblea Nacional del Ecuador (2008): Constitución de la República del Ecuador.

Asamblea Nacional del Ecuador (2009): Ley de Seguridad Pública y del Estado.

Buzan, Barry (2009): The evolution of International Security Studies, New York, Cambridge University Press.

Cabrera, Lester: "Reflexiones en torno a los conceptos de nuevas amenazas, amenazas emergentes y amenazas no tradicionales", Escenarios Actuales, vol. 17, n 1 (2012), pp. 17-26.

Celi, Pablo: "La seguridad multifuncional en la región", en Alda, Sonia y De Sousa, Susana (eds.) (2015): La multidimensionalidad de la seguridad nacional: retos y desafíos de la región para su implementación, Madrid, Instituto Universitario General Gutierrez Mellado, pp. 13-30.

Fuentes, Claudia: "Seguridad Humana: Referencias Conceptuales y Enfoque Práctico para América Latina”, en Rojas, Francisco (ed.) (2012): Seguridad Humana: nuevos enfoques, San José de Costa Rica, FLACSO, pp. 33-54.

García, Bertha: "La gestión de la seguridad y defensa en el Ecuador 2007-2017, bajo un nuevo marco ideológico y geopolítico", en Correa, Loreto (comp.) (2018): Sociedad, Seguridad y Conflicto en América Latina, Ciudad de Panamá, Editorial SIEC, pp. 193-208.

González, Julián: "Libros Blancos de Defensa en Sudamérica: ¿Política exterior o política doméstica?", Revista Brasileira de Estudos de Defensa, vol. 4, nº 2 (2017), pp. 17-48.

González, Mario: "Corte Constitucional deroga las enmiendas constitucionales aprobadas en el 2015", El Comercio, 1 de agosto de 2018, en

https://www.elcomercio.com/actualidad/corteconstitucional-analisis-enmiendas-constitucionderogacion.html

Haro, Patricio (2017): Fuerzas armadas, pretorianismo y calidad de la democracia: Ecuador y Uruguay, Quito, FLACSO Ecuador.

Haro, Patricio: Ecuador: entre la seguridad y la inconstitucionalidad", URVIO - Revista Latinoamericana de Estudios de Seguridad, n 12 (2014), pp. 57-70.

Kacowicz, Arie y David Mares: "Security studies and security in Latin America: the first 200 years", en Mares, David y Kacowicz, Arie (eds.) (2016): Routledge Handbook of Latin American Security, New York, Routledge, pp. 11-30.

Ministerio Coordinador de Seguridad (2011): Plan Nacional de Seguridad Integral 2011-2013.

Ministerio Coordinador de Seguridad (2014): Plan Nacional de Seguridad Integral 2014-2017.

Ministerio Coordinador de Seguridad Interna y Externa (2008): Agenda Nacional de Seguridad Interna y Externa.

Morgan, Patrick (2003): Deterrence Now, Cambridge, Cambridge University Press.

Nunn, Frederick (2011): Relaciones Militares Civiles Sudamericanas en el Siglo XXI, Santiago, Academia de Guerra del Ejército de Chile.

Ordóñez, María Dolores y Cruz, Galo: "La inteligencia militar ecuatoriana en la sociedad del riesgo", URVIO - Revista Latinoamericana de Estudios de Seguridad, n 21 (2017), pp. 56-69.

Orozco, Gabriel: "El concepto de la seguridad en la Teoría de las Relaciones Internacionales", Revista CIDOB d'Afers Internacionals, $\mathrm{n}^{\circ} .72$ (diciembre 2005 - enero 2006), pp. 161-180. 
Pacheco, Guillermo: "Los Libros Blancos: ¿Un paso para consolidar medidas de confianza?”, Revista Ensayos Militares, vol. 2, nº 1 (junio 2016), pp. 101-116.

Paris, Roland: "Human Security. Paradigm Shift or Hot Air?", International Security, vol. 26, n 2 (otoño 2001), pp. 87-102.

Pérez, Diego y Cruz, Galo (2015): Transiciones para la conducción civil y reformas en el sector defensa en Ecuador, Quito, FES-ILDIS.

Pérez, Diego (2018): Liderazgo político y transformaciones institucionales: relaciones Ejecutivo-partidos políticos-Fuerzas Armadas en Ecuador (1996-2006), Quito, Instituto de Altos Estudios Nacionales.

Pérez, Diego: "Fuerzas Armadas en Ecuador. Ideología, políticas públicas y diálogo civil militar", en Namihas, Sandra (edit.) (2018): El Nuevo rol de las Fuerzas Armadas en Bolivia, Brasil, Chile, Colombia, Ecuador y Perú. Red de Política de Seguridad, Lima, Pontificia Universidad Católica del Perú - Konrad Adenauer Stiftung, pp. 101-138.

Piedra, Lorena (2012): Coyunturas críticas en los sistemas de inteligencia, Quito, FLACSOEcuador.

Redacción Ecuavisa: "Militares se unirán a nuevos grupos de seguridad". Ecuavisa, 2 de diciembre de 2013, en https://www.ecuavisa.com/articulo/noticias/actualidad/47650-militaresse-unirian-nuevos-grupos-seguridad

Redacción Plan V: "El legado del correato en armamento y gasto militar". Plan V, 3 de septiembre de 2018, en http://www.planv.com.ec/historias/sociedad/el-legado-del-correatoarmamento-y-gasto-militar

Redacción Política: "En video, Alto Mando pide a soldados evitar rumores sobre recorte en FF.AA.", El Universo, 12 de diciembre de 2013, en https:/www.eluniverso.com/noticias/2013/12/12/nota/1908226/video-alto-mando-pidesoldados-evitar-rumores-sobre-recorte-ffaa

Redacción Política: "Ricardo Patiño reitera que se busca justicia en pago de pensiones militares”. El Universo, 18 de julio de 2016, en https://www.eluniverso.com/noticias/2016/07/18/nota/5698200/ricardo-patino-reitera-que-nose-incrementaran-pensiones-que-esten

Rivera, Fredy (2012): La seguridad perversa: Política, democracia y derechos humanos en el Ecuador 1998-2006, Quito, FLACSO.

Rivera, Fredy (2016): "Inteligencia estratégica e inteligencia política: los claro-oscuros del caso ecuatoriano", en Inteligencia Estratégica Contemporánea, Sangolquí, ESPE, pp. 133-148.

Rivera, Fredy: "Plan Ecuador: oportunidad perdida, oportunidad posible", en Guzmán, Ivonne (ed.) (2018): El Contagio. Fin de la Isla de Paz, Quito, Mediato, pp. 87-110.

Saint-Pierre, Hector: "'Defense 'or 'Security'? reflections on concepts and ideologies", Contexto Internacional, vol. 33, $\mathrm{n}^{\mathrm{o}} 2$ (2011), pp. 407-433.

Stepan, Alfred (1988): Rethinking Military Politics: Brazil and the Southern Cone, Princeton, Princeton University Press.

Thies, Cameron: “Traditional security: war and peace", en Mares, David y Kacowicz, Arie (eds.) (2016): Routledge Handbook of Latin American Security, New York, Routledge, pp. 113126. 
Vitelli, Marina: "América del Sur: de la seguridad cooperativa a la cooperación disuasoria", Foro Internacional, vol. 56, nº 3 (julio-septiembre 2016), pp. 724-755.

Zepeda, Beatriz: "La Política Exterior del Ecuador Durante el Gobierno de Rafael Correa: Un Balance", en Mathieu, Hans y Niño, Catalina (eds.) (2011): Anuario 2011 de la Seguridad Regional en América Latina y El Caribe, Quito, Friedrich Ebert Stiftung, pp. 114-126. 